\section{Efficacy of olanzapine combined with valproate or lithium in the treatment of dysphoric mania}

\author{
ROBERT W. BAKER, EILEEN BROWN, HAGOP S. AKISKAL, \\ JOSEPH R. CALABRESE, TERENCE A. KETTER, LESLIE M. SCHUH, \\ PAULA T. TRZEPACZ, JOHN G. WATKIN and MAURICIO TOHEN
}

\section{Background Few controlled studies examine the treatment of depressive features in mania.}

\begin{abstract}
Aims To evaluate the efficacy of olanzapine, in combination with lithium or valproate, for treating depressive symptoms associated with mania.
\end{abstract}

Method Secondary analysis of a 6-week, double-blind, randomised study of olanzapine (5-20 mg/day) or placebo combined with ongoing valproate or lithium open treatment for 344 patients in mixed or manic episodes. This analysis focused on a dysphoric subgroup with baseline Hamilton Rating Scale for Depression (HRSD) total scores of 20 or over contrasted with non-dysphoric patients.

\section{Results In the dysphoric subgroup $(n=85)$ mean HRSD total score improvement was significantly greater in olanzapine co-therapy patients than in those receiving placebo plus lithium or valproate $(P<0.001)$. Substantial contributors to this superiority included the HRSD Maier sub-scale $(P=0.013)$ and the suicide item $(P=0.001)$. Total Young Mania Rating Scale improvement was also superior with olanzapine co-therapy. \\ Conclusions In patients with acute dysphoric mania, addition of olanzapine to ongoing lithium or valproate monotherapy significantly improved depressive symptom, mania and suicidality ratings.}

Declaration of interest R.W.B., E.B., L.M.S., P.T.T., J.G.W. and M.T. are employees of Eli Lilly and Company.
Despite the fact that dysphoric symptoms represent a significant complicating factor in the successful treatment of bipolar disorder, controlled trials regarding the depressive aspects of acute mania are rare (Montgomery et al, 2000). A post hoc analysis of pooled data from two double-blind, placebo-controlled trials supported the efficacy of olanzapine monotherapy for both manic and depressive aspects of dysphoric mania (Baker et al, 2003), although the decrease in scores on the Hamilton Rating Scale for Depression (HRSD; Hamilton, 1967) was driven by improvement in items related to sleep, insight and paranoia. A randomised double-blind, placebo-controlled study demonstrated the efficacy of olanzapine co-therapy in patients with bipolar disorder who had inadequate responses to valproate or lithium monotherapy (Tohen et al, 2002). We describe a secondary analysis of data from that study, assessing both depression and manic treatment responses among dysphoric and non-dysphoric patients with bipolar I disorder, who received either olanzapine or placebo in addition to lithium or valproate.

\section{METHOD}

\section{Study design}

Data were derived from a large, multicentre double-blind study. Patients whose symptoms of mania were partially nonresponsive to 2 weeks of treatment with valproate or lithium monotherapy at therapeutic serum levels were randomised to receive either adjunctive olanzapine (hereafter referred to as 'combination therapy') or adjunctive placebo ('monotherapy') in addition to their existing lithium or valproate monotherapy. We report secondary post hoc analyses focusing on a subgroup of patients with mania or mixed disorder who had substantial comorbid depression at baseline, and examine whether therapeutic response in this 'dysphoric' group differed from the remaining 'non-dysphoric' patients. The study was conducted at 33 sites in the USA and 5 sites in Canada. Detailed methods and the primary results of this study have been published by Tohen et al (2002).

\section{Patients}

The protocol was approved by the institutional review board at each site, and all patients provided written informed consent prior to administration of any study procedures or study medications. At enrolment, all patients had bipolar I disorder as defined by DSM-IV criteria (American Psychiatric Association, 1994), manic or mixed episode, with or without psychotic features, assessed independently of the symptom rating scale scores. Diagnosis was confirmed using the patient version of the Structured Clinical Interview for DSM-IV (SCID-P; First et al, 1997). Patients were required to have had at least two previous depressed, manic or mixed episodes, and to have had documented treatment with lithium or valproate for at least 2 weeks prior to visit 1 , with a therapeutic blood level of lithium $(0.6-1.2 \mathrm{mmol} / \mathrm{l})$ or valproate $(50-125 \mu \mathrm{g} /$ $\mathrm{ml}$ ) at visit 1 . Patients were included in the study only if they showed inadequate response to monotherapy for the 2 weeks prior to randomisation, evidenced by a total score of 16 or more on the Young Mania Rating Scale (YMRS; Young et al, 1978). After meeting entry criteria, patients were randomised in a 2:1 ratio to receive either adjunctive olanzapine (flexible daily dosage of 5,10 or $20 \mathrm{mg}$ ) or adjunctive placebo in conjunction with their current valproate or lithium for 6 weeks. Olanzapine was initiated at a daily dosage of $10 \mathrm{mg}$. Plasma levels of lithium or valproate were documented to be within their therapeutic serum ranges.

Patients were also permitted adjunctive benzodiazepine ( $\leqslant 2 \mathrm{mg}$ daily of lorazepam equivalents) for no more than 14 days cumulatively. Aside from the study drugs, benzodiazepines and anticholinergics (benzatropine mesilate, $\leqslant 2 \mathrm{mg}$ per day), no other psychotropic drug was permitted during the study.

\section{Assessments}

Severity of illness was measured by the 11-item YMRS and the 21-item HRSD. Ratings were completed at baseline and weekly during therapy. The post boc analysis focused on patients with moderate 
to severe dysphoric mania, as defined by a baseline HRSD total score of 20 or over. This threshold has been used by others to stratify patients into mild $v$. moderate to severely ill categories in depression studies (Thase et al, 1997). Changes in severity of depressive symptoms were assessed using the HRSD total score, HRSD Maier subscale and HRSD individual items. The Maier sub-scale comprises six items: item 1 (depressed mood), item 2 (guilt), item 7 (work and activities), item 8 (retardation), item 9 (agitation) and item 10 (psychic anxiety) (Maier et al, 1985).

\section{Statistical method}

Comparisons of baseline demographic and illness characteristics between patients with a baseline HRSD total score of 20 or above and patients with a score below 20 were performed using analysis of variance (ANOVA) with categorisation (dysphoric at baseline/non-dysphoric at baseline) in the model for continuous variables and using Fisher's exact test for categorical variables. Descriptive baseline demographic factors for the olanzapine and placebo treatment groups were also tabulated. The primary efficacy measure was the mean change from baseline to end-point (last observation carried forward up to week 6) for HRSD total score. The ANOVA was performed with treatment, dysphoric/nondysphoric categorisation and interaction between categorisation and treatment in the model. An additional model with treatment, dysphoric/non-dysphoric categorisation, interaction between categorisation and treatment, mood stabiliser (valproate or lithium) and the interaction between mood stabiliser and categorisation was also explored. The hypothesis that mean improvement in HRSD score in the olanzapine combination therapy group over the placebo monotherapy group did not differ between patients with and without baseline depressive symptoms was tested with the interaction test of dysphoric/non-dysphoric categorisation by treatment. Similar analyses were performed for the YMRS total score. Effect sizes were calculated as the difference between combination therapy and monotherapy divided by the estimated standard deviation obtained from the square root of the mean square error of the ANOVA model.

To evaluate the time course of improvement, a mixed-model repeated measures (MMRM) analysis of variance was fitted for change from baseline in HRSD total score with investigator, treatment, visit, visit-treatment interaction, dysphoric categorisation (dysphoric $v$. non-dysphoric), dysphoric categorisation-treatment and dysphoric categorisation-visit and the threeway interaction of dysphoric categorisationtreatment-visit. An unstructured covariance matrix was fitted to the within-patient repeated measures. Using contrasts within the repeated measures model, the main overall effect of treatment within each dysphoric subgroup was assessed, as was the change from baseline to each visit between treatment groups.

Patients were analysed on an intentionto-treat basis for all analyses. Patients with a baseline assessment and at least one postbaseline measurement were included in the analysis. The YMRS and HRSD total scores were derived from the individual items; if any single item was missing, the total score was treated as missing. All $P$ values were based on two-tailed tests with a significance level of 0.05 .

\section{RESULTS}

\section{Patients}

Baseline patient and illness characteristics by dysphoric/non-dysphoric categorisation and treatment group are summarised in Table 1. Of 344 randomised patients, 85 met the study criterion for dysphoric mania (olanzapine combination treatment, $n=60$; placebo monotherapy, $n=25$ ) and 259 for non-dysphoric mania (combination treatment, $n=169$; monotherapy, $n=90$ ). Within the dysphoric subgroup, $84.7 \%$ of patients met DSM-IV criteria for a mixed episode. The dysphoric and non-dysphoric groups contained similar proportions of patients being treated with valproate (70.6\% and $64.3 \%$ respectively, $P=0.356$ ) and lithium $(29.4 \%$ and $35.7 \%$ respectively). Mean modal dose of olanzapine did not differ significantly between dysphoric and non-dysphoric patients $(11.4 \mathrm{mg} v$. $10.0 \mathrm{mg} ; P=0.15$ ).

\section{Efficacy: depressive symptoms}

For the analysis of change in HRSD total score from baseline to end-point, the interaction between mood stabiliser (valproate or lithium) and dysphoric categorisation was not significant $(P=0.290)$, indicating that the treatment effect of olanzapine combination therapy over monotherapy was not dependent upon whether patients were taking valproate or lithium. In addition, the main effect of mood stabiliser was also not significant $(P=0.528)$, indicating that change in HRSD score did not differ in patients taking valproate $v$. those taking lithium. Since these two terms were not statistically significant, they were dropped from the model for the analysis of HRSD total score as well as the HRSD Maier sub-scale and individual items.

For the HRSD total score, there was a statistically significant interaction between categorisation (baseline dysphoric/ non-dysphoric) and treatment group $(P=0.016)$, indicating that mean HRSD score improvement in combination therapy patients over monotherapy patients differed between those with and without baseline dysphoria (Table 2). In both dysphoric and non-dysphoric patients, improvement in HRSD total score was significantly greater for patients receiving combination therapy compared with monotherapy (dysphoric group $-8.82 v$. $-1.42, P<0.001$; non-dysphoric group $-2.87 \quad v .0 .01$, $P=0.002$ ). However, the magnitude of the difference between combination and monotherapy treatment groups was larger for the dysphoric patients (Fig. 1), thus driving the significant interaction. The difference between treatment groups is also apparent from a comparison of effect sizes for the dysphoric patients (1.11) with that of non-dysphoric patients $(0.43)$.

A significant interaction existed between treatment and dysphoric subgroup, indicating that treatment group differences were dependent upon patients' dysphoric status (dysphoric $v$. non-dysphoric). Furthermore, MMRM analysis detected a significant interaction between treatment and visit, indicating that the response over time differed between the treatment groups $(P=0.009)$. Plots of changes in mean HRSD total scores over time for dysphoric and non-dysphoric subgroups are presented in Fig. 1. For each subgroup, an overall treatment difference was detected (olanzapine combination therapy superior to monotherapy; $P<0.001$ in both cases) and this difference was observed as early as the first post-randomisation visit (week 1).

Analysis of the HRSD Maier sub-scale revealed no significant interaction between dysphoric categorisation and treatment, indicating that the beneficial effect of combination therapy over monotherapy was similar in dysphoric and non-dysphoric patients. In both groups, improvement in Maier sub-scale score was significantly 
Table I Baseline patient and illness characteristics

\begin{tabular}{|c|c|c|c|c|c|c|c|}
\hline \multirow[t]{2}{*}{ Characteristic } & \multicolumn{3}{|c|}{ Baseline dysphoric mood' } & \multicolumn{3}{|c|}{ No baseline dysphoric mood' } & \multirow[t]{2}{*}{$P^{4}$} \\
\hline & $\begin{array}{l}\text { Combination therapy }{ }^{2} \\
\qquad(n=60)\end{array}$ & $\begin{array}{c}\text { Monotherapy }{ }^{3} \\
(n=25)\end{array}$ & $\begin{array}{c}\text { Total } \\
(n=85)\end{array}$ & $\begin{array}{l}\text { Combination therapy }{ }^{2} \\
\qquad(n=169)\end{array}$ & $\begin{array}{c}\text { Monotherapy }{ }^{3} \\
(n=90)\end{array}$ & $\begin{array}{c}\text { Total } \\
(n=259)\end{array}$ & \\
\hline Age, years: mean (s.d.) & $38.16(10.33)$ & $40.28(11.20)$ & $38.78(10.57)$ & $41.64($ (II.44) & $40.46(10.78)$ & $41.23(11.20)$ & 0.077 \\
\hline \multicolumn{8}{|l|}{ Gender } \\
\hline Male (\%) & 37 & 28 & 34 & 47 & 63 & 53 & 0.004 \\
\hline \multicolumn{8}{|l|}{ Ethnicity } \\
\hline White (\%) & 82 & 96 & 86 & 87 & 81 & 85 & 1.000 \\
\hline \multicolumn{8}{|l|}{ Episode type } \\
\hline Mixed (\%) & 85 & 84 & 85 & 44 & 37 & 41 & $<0.001$ \\
\hline Psychotic features present $(\%)^{6}$ & 48 & 36 & 45 & 27 & 33 & 29 & 0.012 \\
\hline Rapid cycling (\%) ${ }^{7}$ & 52 & 40 & 48 & 42 & 48 & 44 & 0.531 \\
\hline \multicolumn{8}{|l|}{ Mood stabiliser } \\
\hline Valproate (\%) & 63 & 88 & 71 & 68 & 57 & 64 & 0.356 \\
\hline \multicolumn{8}{|c|}{ Previous depression lifetime episodes (\%) } \\
\hline$\leqslant 2$ & 10 & 12 & II & 26 & 25 & 26 & $0.001^{8}$ \\
\hline $3-10$ & 30 & 44 & 34 & 36 & 27 & 33 & \\
\hline II-50 & 20 & 12 & 18 & 19 & 26 & 22 & \\
\hline$>50$ & 40 & 32 & 38 & 19 & 23 & 20 & \\
\hline \multicolumn{8}{|c|}{ Previous mania lifetime episodes (\%) } \\
\hline$\leqslant 2$ & 13 & 16 & 14 & 20 & 13 & 18 & $0.011^{8}$ \\
\hline $3-10$ & 22 & 44 & 28 & 32 & 37 & 34 & \\
\hline$I I-50$ & 23 & 4 & 18 & 25 & 19 & 23 & \\
\hline$>50$ & 42 & 36 & 40 & 22 & 31 & 25 & \\
\hline \multicolumn{8}{|c|}{ Previous mixed lifetime episodes (\%) } \\
\hline$\leqslant 2$ & 22 & 32 & 25 & 46 & 47 & 47 & $<0.001^{8}$ \\
\hline $3-10$ & 20 & 20 & 20 & 21 & 13 & 18 & \\
\hline $1 \mathrm{II}-50$ & 15 & 8 & 13 & 12 & 16 & 13 & \\
\hline$>50$ & 42 & 40 & 42 & 20 & 25 & 22 & \\
\hline YMRS total score, mean (s.d.) & $23.80(5.16)$ & $23.24(4.65)$ & $23.64(4.99)$ & $21.81(5.33)$ & $22.57(5.30)$ & $22.07(5.32)$ & 0.018 \\
\hline HRSD total score, mean (s.d.) & $25.33(4.82)$ & $24.60(4.12)$ & $25.18(4.62)$ & $10.44(5.39)$ & $10.39(5.06)$ & $10.42(5.27)$ & $<0.001$ \\
\hline
\end{tabular}

HRSD, Hamilton Rating Scale for Depression; YMRS, Young Mania Rating Scale.

I. Baseline dysphoric mood defined as an HRSD total score $\geqslant 20$.

2. Olanzapine plus lithium or valproate.

3. Placebo plus lithium or valproate.

4. Statistical tests performed between total columns and not between treatment groups; means analysed using a type III sum of squares analysis of variance with categorisation in model and frequencies analysed using Fisher's exact test.

5. Mixed type was defined by investigators using DSM-IV criteria, independently of mood rating scale scores.

6. Of the patients who showed psychotic features, $85.5 \%$ of the dysphoric individuals and $76.3 \%$ of the non-dysphoric were mood congruent.

7. Rapid cycling defined as four or more manic, depressed or mixed episodes in the previous year.

8. Cochran-Armitage trend test.

greater for patients receiving olanzapine combination therapy than for those receiving monotherapy (dysphoric group -2.73 v. $-0.45, P=0.013$; non-dysphoric group -1.07 v. $0.59, P=0.001$ ).

Statistically significant interactions between dysphoric categorisation and treatment were observed for five HRSD individual items (Table 2). For each of these five items, dysphoric patients receiving olanzapine combination therapy demonstrated significantly superior improvement over those receiving monotherapy, corresponding to large effect sizes.
In contrast, patients in the non-dysphoric category exhibited no significant difference for these five items and small effect sizes between treatment groups. In the case of item 10 (psychic anxiety), significant between-treatment differences were observed in both dysphoric and nondysphoric patient groups. For HRSD items 1 (depressed mood) and 2 (feelings of guilt), effect sizes were approximately 0.45 for both subgroups - however, significant treatment differences were only seen in the non-dysphoric group, possibly owing to the smaller size of the dysphoric group.
In the case of HRSD items 20 (paranoid symptoms) and 21 (obsessional and compulsive symptoms), significant differences were detected between combination and monotherapy in the dysphoric patient group, whereas in the non-dysphoric group there was a significant difference between treatment groups for item 20 only.

\section{Efficacy: manic symptoms}

Analysis of YMRS total score revealed no significant interaction between mood stabiliser (valproate or lithium) and 
Table 2 Summary of efficacy measures: least square means from analysis of variance model

\begin{tabular}{|c|c|c|c|c|c|c|c|c|c|}
\hline \multirow[t]{2}{*}{ Item } & \multirow{2}{*}{$\begin{array}{c}\text { Interaction' } \\
P\end{array}$} & \multicolumn{4}{|c|}{ Dysphoric patients } & \multicolumn{4}{|c|}{ Non-dysphoric patients } \\
\hline & & $\begin{array}{c}\text { Combination } \\
\text { therapy }^{2}\end{array}$ & Monotherapy ${ }^{3}$ & $P^{4}$ & Effect size & $\begin{array}{c}\text { Combination } \\
\text { therapy }^{2}\end{array}$ & Monotherapy ${ }^{3}$ & $P^{4}$ & Effect size \\
\hline \multicolumn{10}{|l|}{ HRSD } \\
\hline Total & 0.016 & -8.82 & -1.42 & $<0.001$ & I.II & -2.87 & 0.01 & 0.002 & 0.43 \\
\hline Maier sub-scale & 0.556 & -2.73 & -0.45 & 0.013 & 0.61 & -1.07 & 0.59 & 0.001 & 0.45 \\
\hline \multicolumn{10}{|l|}{ Individual items } \\
\hline I Depressed mood & 0.817 & -0.31 & 0.15 & 0.111 & 0.39 & -0.04 & 0.50 & $<0.001$ & 0.45 \\
\hline 2 Feelings of guilt & 0.950 & -0.55 & -0.14 & 0.065 & 0.45 & -0.34 & 0.09 & $<0.001$ & 0.47 \\
\hline 3 Suicide & 0.016 & -0.45 & 0.15 & 0.001 & 0.80 & 0.07 & 0.16 & 0.361 & 0.12 \\
\hline 4 Insomnia (early) & 0.048 & -0.90 & -0.18 & 0.003 & 0.73 & -0.33 & -0.16 & 0.182 & 0.18 \\
\hline 5 Insomnia (middle) & $<0.001$ & -0.89 & 0.09 & $<0.00 \mathrm{I}$ & 1.13 & -0.45 & -0.33 & 0.322 & 0.13 \\
\hline 6 Insomnia (late) & 0.040 & -0.75 & -0.16 & 0.005 & 0.69 & -0.35 & -0.25 & 0.416 & 0.11 \\
\hline 7 Work and activities & 0.933 & -0.38 & -0.27 & 0.686 & 0.12 & -0.02 & 0.12 & 0.367 & 0.14 \\
\hline 8 Retardation & 0.333 & -0.10 & 0.07 & 0.275 & 0.27 & 0.18 & 0.17 & 0.960 & -0.01 \\
\hline 9 Agitation & 0.631 & -0.76 & -0.41 & 0.181 & 0.33 & -0.56 & -0.36 & 0.158 & 0.19 \\
\hline 10 Anxiety (psychic) & 0.152 & -0.63 & 0.15 & 0.003 & 0.74 & -0.30 & 0.05 & 0.013 & 0.34 \\
\hline II Anxiety (somatic) & 0.322 & -0.50 & -0.17 & 0.132 & 0.37 & -0.14 & -0.06 & 0.512 & 0.09 \\
\hline I2 Somatic symptoms (GI) & 0.031 & -0.42 & -0.09 & 0.008 & 0.65 & -0.11 & -0.09 & 0.775 & 0.04 \\
\hline I3 Somatic symptoms (general) & 0.992 & -0.12 & -0.02 & 0.584 & 0.13 & 0.02 & 0.13 & 0.314 & 0.14 \\
\hline I4 Genital symptoms & 0.110 & -0.15 & 0.14 & 0.121 & 0.38 & 0.07 & 0.02 & 0.594 & -0.07 \\
\hline I5 Hypochondriasis & 0.412 & -0.49 & -0.30 & 0.266 & 0.27 & 0.02 & 0.04 & 0.768 & 0.04 \\
\hline 16 Loss of weight & 0.565 & 0.00 & 0.13 & 0.128 & 0.37 & -0.01 & 0.06 & 0.122 & 0.21 \\
\hline 17 Insight & 0.845 & 0.03 & -0.04 & 0.590 & -0.13 & -0.08 & -0.12 & 0.573 & -0.08 \\
\hline I8 Diurnal variation & 0.237 & -0.35 & -0.43 & 0.653 & -0.11 & -0.09 & 0.06 & 0.099 & 0.22 \\
\hline 21 OC symptoms & 0.233 & -0.11 & 0.16 & 0.033 & 0.52 & -0.11 & -0.02 & 0.171 & 0.19 \\
\hline YMRS Total & 0.111 & -11.75 & -4.65 & $<0.001$ & 0.84 & -13.06 & -9.78 & 0.005 & 0.39 \\
\hline
\end{tabular}

Gl, gastrointestinal; HRSD, Hamilton Rating Scale for Depression; OC, obsessional and compulsive; YMRS, Young Mania Rating Scale.

I. Interaction between dysphoric categorisation and treatment.

2. Olanzapine plus lithium or valproate.

3. Placebo plus lithium or valproate.

4. Combination $v$. monotherapy treatment groups.

treatment, or in the main effect of mood stabiliser $(P>0.20$ for both), indicating that the difference in treatment group response was not dependent upon whether patients were receiving valproate or lithium. Furthermore, no statistically significant interaction existed between dysphoric/non-dysphoric categorisation and treatment $(P=0.111$; Table 2$)$, indicating that improvements in mania severity for combination therapy over monotherapy were independent of dysphoric/non-dysphoric categorisation. Statistically significant differences were detected between treatment groups for both dysphoric and nondysphoric categories. The difference between treatment groups was numerically (although not significantly) larger for the dysphoric patients (interaction $P=0.111$ ).
However, this was due primarily to a larger monotherapy response among non-dysphoric patients (mean change for dysphoric patients $-4.65 v$. non-dysphoric patients -9.78 ; $P=0.013)$ rather than a significant difference in combination treatment response between groups (mean change for dysphoric patients $-11.75 v$. non-dysphoric patients $-13.06 ; P=0.361$ ).

\section{DISCUSSION}

This analysis found that olanzapine therapy combined with either valproate or lithium effectively treated coexisting manic and depressive symptoms in patients who experienced inadequate response or breakthrough symptoms during lithium or valproate monotherapy. Patients receiving combination treatment demonstrated significantly greater improvement compared with those receiving monotherapy in depressive symptom severity (HRSD total score). A substantial proportion of the treatment benefits in depression occurred within core mood symptoms. Significant improvements were also observed in mania symptom severity (YMRS total score) in the combination therapy group compared with the monotherapy group.

Although defined rather differently, the terms 'dysphoric mania', 'depressive mania' and 'mixed mania' are used somewhat interchangeably, as each describes a manic episode complicated by prominent comorbid depressive features (Bauer et al, 1994; Akiskal et al, 1998; Dilsaver et al, 1999; 


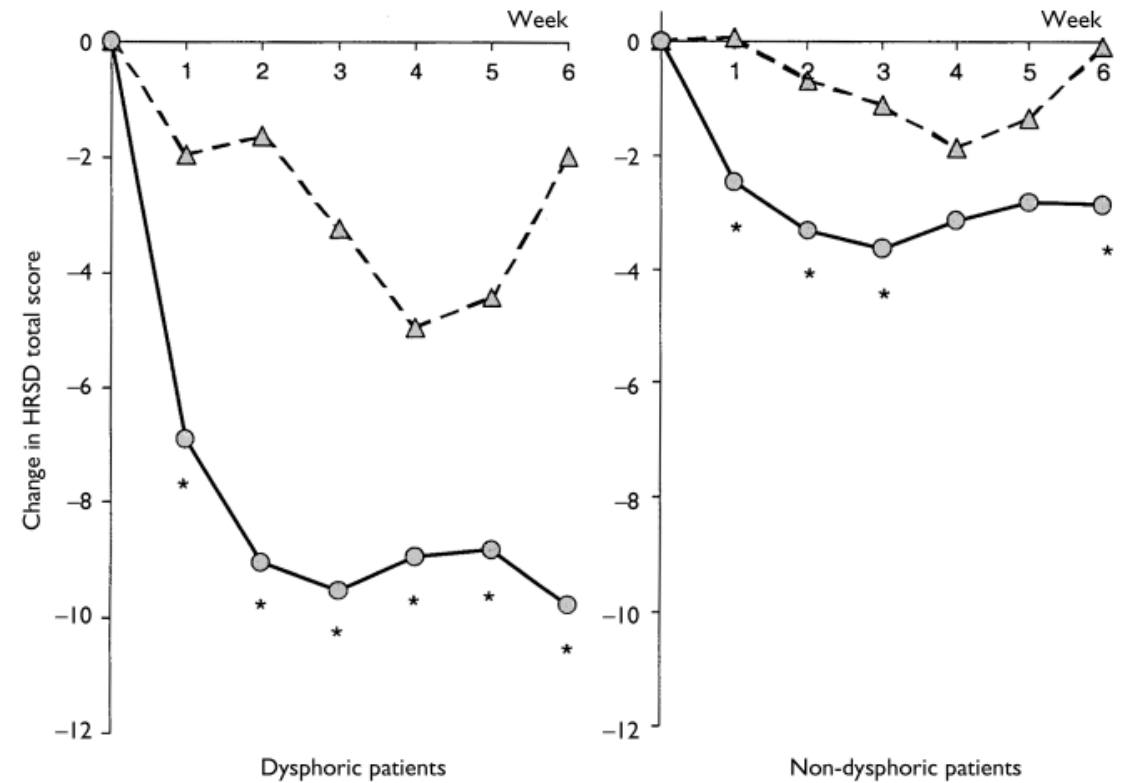

Fig. I Mean change in Hamilton Rating Scale for Depression (HRSD) total score for dysphoric patients (lefthand graph) and non-dysphoric patients (right-hand graph) receiving olanzapine plus lithium or valproate (combination therapy; circles) or placebo plus lithium or valproate (monotherapy; triangles). Dysphoric patients: combination therapy $n=60$, monotherapy $n=25$; non-dysphoric patients: combination therapy $n=169$, monotherapy $n=90 ; *, P<0.01$ v. placebo.

Perugi et al, 2001). Given that patients experiencing these dysphoric manic episodes are reported to constitute up to two-thirds of bipolar disorder clinical samples (Akiskal et al, 2000), it might be expected that effective treatment options would exist. However, the overall prognosis for patients with dysphoric mania is worse than for those with pure mania (Himmelhoch \& Garfinkel, 1986; Keller et al, 1986; Secunda et al, 1987), and in fact our data at baseline (see Table 1) indicate greater severity of many variables in the dysphoric group.

A key treatment challenge in bipolar disorder, especially among patients with mixed mania and rapid cycling, is to bring about improvement of symptoms of one mood pole without worsening or accelerating the onset of symptoms of the opposite pole. Ideally, one medication would be employed to treat both mood symptom poles simultaneously. However, so far controlled monotherapy studies have failed to demonstrate that this goal has been achieved, especially in mixed or dysphoric mania. Among the classic mood stabilisers, the body of evidence suggesting antidepressant effects during lithium monotherapy is much less robust than that supporting its antimanic properties, and valproate may be preferable to lithium in the treatment of dysphoric mania (McElroy et al, 1988;
Freeman et al, 1992; Swann et al, 1997). For valproate, evidence of efficacy is stronger for manic features than for depressive features of dysphoric mania (Swann et al, 1997). Olanzapine's antimanic properties are well established (Tohen et al, 2000). In addition, olanzapine appears to reduce symptoms of bipolar depression, albeit less effectively when given alone than in combination with fluoxetine (Tohen et al, 2003a). In an earlier study we described the efficacy of olanzapine monotherapy in treating both manic and depressive symptoms in dysphoric and non-dysphoric in-patients (Baker et $a l, 2003)$. Olanzapine demonstrated significant improvement in YMRS total score compared with placebo in both dysphoric and non-dysphoric patients, and also produced significant improvement in HRSD total score in the dysphoric group (Baker et al, 2003). This is to our knowledge the only previous placebo-controlled demonstration of simultaneous improvement in depressive and manic symptoms of dysphoric mania during treatment with a single agent. Further study seemed appropriate, however, given that the previous report had important limitations, such as small sample size and non-statistically significant improvement in Maier sub-scale scores.

Our analysis includes effect size calculations. An effect size of 1.0 reflects difference between treatment groups of the same magnitude as 1 standard deviation within the population. This can be translated into a probability statement that indicates that the improvement in score of an average individual in the comparison group exceeds that of $84 \%$ of patients in the placebo group. Effect size is a useful tool for comparing findings across studies, and by convention an effect size of 0.2 is considered small, 0.5 moderate and 0.8 or greater large (Cohen, 1988). This study demonstrated a large effect size (1.11) for improvement of depression in patients with baseline dysphoria who received olanzapine in addition to lithium or valproate. Whereas our previous report did not demonstrate clear benefit in terms of Maier core mood sub-scale, this study demonstrated a moderate effect size for this subscale $(0.61)$.

Our analyses have several limitations. First, these are post hoc analyses of data from a clinical trial which evaluated severity of manic symptoms (YMRS total score) as the primary efficacy measure. Second, assignment to valproate or lithium was not randomised but reflected the treatment preferences of clinicians and investigators. Third, the study design lacked an olanzapine monotherapy arm, which makes it difficult to draw any conclusions regarding the relative contributions of olanzapine alone, or synergistic effects with the concomitant medication, to the overall treatment benefits. In fact, no inference can be drawn regarding the relative merit of other treatments or combinations that were not evaluated in this trial. For example, improvement on classical antipsychotic agents would be of interest, given that a recent mania treatment comparison of olanzapine and haloperidol did not establish differential treatment response of depressive symptoms among the small $(n=19)$ subgroup with baseline HRSD scores of 20 or over (Tohen et al, 2003b). Fourth, the high proportion of participants in rapid-cycling disorder is notable. A rapid-cycling course may affect mania phenomenology - for example, some 'dysphoric' patients may be in transition from mania to depression (Kraepelin, 1921; Himmelhoch et al, 1976; McElroy et al, 1992) - and its relationship to dysphoric mania is unclear (Himmelhoch et al, 1976; Post et al, 1989; McElroy et al, 1992). Finally, the subject group of interest (patients with a baseline HRSD total score of 20 or above) was not defined a priori. In this study most dysphoric patients were 
diagnosed with mixed episodes, but interestingly there was imperfect homology between a DSM-IV mixed mania diagnosis and dysphoric mania as defined by rating scales. The DSM-IV definition of a mixed manic phase requires patients to simultaneously meet criteria for both a major depressive episode and a manic episode. However, some argue that a DSM-IV syndromal approach may not be optimal for identifying patients with mania and clinically important comorbid depressive features (Cassidy et al, 1998; Cassidy \& Carroll, 2001; Dilsaver et al, 1999), and a number of proposals have been put forward which attempt to provide a framework within which a diagnosis of dysphoric mania may be made (McElroy et al, 1992; Perugi et al, 1997; Cassidy et al, 2000; Akiskal et al, 2003). These proposals require the presence of a full manic episode, plus the presence of two or three defined depressive symptoms. For the purpose of this study, in which all patients met criteria for a manic episode, we used a threshold HRSD total score of 20 or over to define a group of patients displaying prominent depressive features. We believe that this use of an objective rating scale represents a clinically meaningful approach to identifying subjects. In the context of this clinical trial, the DSM-IV and HRSD categorisation systems are somewhat incongruent, with approximately twice the number of patients being identified as 'mixed' as were categorised as dysphoric based on symptom ratings. One potential contribution to the incongruence is that symptom ratings coincided with treatment randomisation, whereas diagnostic verification could have taken place up to a week prior to randomisation. In any case, the dysphoric subgroup primarily included patients with diagnosis of a mixed episode.

In conclusion, our analyses found that olanzapine in combination with either lithium or valproate was effective in improving the severity of depressive symptoms coexisting with acute mania. Furthermore, olanzapine co-therapy with lithium or valproate also demonstrated efficacy in the management of manic symptoms, irrespective of whether such symptoms were complicated by concurrent dysphoria. Given the prognostic implications of dysphoric features in mania, and the limited number of pharmacological options currently available for such patients, prospectively designed studies of combination

\section{CLINICAL IMPLICATIONS}

Although dysphoric symptoms represent a significant complicating factor in the successful treatment of bipolar disorder, current treatment options are limited.

- In this study, olanzapine in combination with either lithium or valproate was effective in improving the severity of depressive symptoms coexisting with acute mania.

- Olanzapine co-therapy also demonstrated efficacy in managing manic symptoms, irrespective of the presence of concurrent dysphoria.

\section{LIMITATIONS}

- The study used post hoc analysis of data from a clinical trial which evaluated mania rating scale score as the primary efficacy measure.

Assignment to valproate or lithium therapy was not randomised but reflected the treatment preferences of clinicians and investigators.

The study did not include a typical antipsychotic comparator.

ROBERT W. BAKER, MD, Lilly Research Laboratories, Eli Lilly \& Co., Indianapolis, Indiana, and Department of Psychiatry and Human Behavior, University of Mississippi, Jackson, Mississippi; ElLEEN BROWN, PhD, Lilly Research Laboratories, Eli Lilly \& Co., Indianapolis, Indiana; HAGOP S. AKISKAL, MD, International Mood Center, Department of Psychiatry, University of California at San Diego, San Diego, California; JOSEPH R. CALABRESE, MD, Department of Psychiatry, Case Western Reserve University, University Hospitals of Cleveland, Cleveland, Ohio; TERENCE A. KETTER, MD, Department of Psychiatry and Behavioral Sciences, Stanford University, Stanford, California; LESLIE M. SCHUH, PhD, Lilly Research Laboratories, Eli Lilly \& Co., Indianapolis, Indiana; PAULA T. TRZEPACZ, MD, Lilly Research Laboratories, Eli Lilly \& Co., Indianapolis, Indiana, Department of Psychiatry and Human Behavior, University of Mississippi, Jackson, Mississippi, and Department of Psychiatry, Tufts University Medical School, Boston, Massachusetts; JOHN G.WATKIN, DPhil, Lilly Research Laboratories, Eli Lilly \& Co., Indianapolis, Indiana; MAURICIOTOHEN, MD, DrPH, Lilly Research Laboratories, Eli Lilly \& Co., Indianapolis, Indiana, and Department of Psychiatry, Harvard Medical School/ McLean Hospital, Belmont, Massachusetts, USA

Correspondence: Dr Robert Baker, Lilly Research Laboratories, Lilly Corporate Center, Indianapolis, IN 46285, USA. Fax: +317 276 7100; e-mail: robertbaker@lilly.com

(First received IO November 2003, final revision 5 August 2004, accepted II August 2004)

therapy with mood stabilisers and typical antipsychotics in the management of dysphoric mania are encouraged. Such studies should consider innovative strategies for defining dysphoric mania that transcend the limited perspective of DSM-IV mixed states (Perugi et al, 1997; Akiskal et al, 2003).

\section{ACKNOWLEDGEMENT}

This work was supported by Lilly Research Laboratories.

\section{REFERENCES}

Akiskal, H. S., Hantouche, E. G., Bourgeois, M. L., et al (1998) Gender, temperament, and the clinical picture in dysphoric mixed mania: findings from a French national study (EPIMAN). Journal of Affective Disorders, 50, $175-186$

Akiskal, H. S., Bourgeois, M. L., Angst, J., et al (2000) Re-evaluating the prevalence of and diagnostic composition within the broad clinical spectrum of bipolar disorders. Journal of Affective Disorders, 59 (suppl. I), S5-S30.

Akiskal, H. S., Azorin, J. M. \& Hantouche, E. G. (2003) Proposed multidimensional structure of mania: beyond the euphoric-dysphoric dichotomy. Journal of Affective Disorders, 73, 7-18.

American Psychiatric Association (1994) Diagnostic and Statistical Manual of Mental Disorders (4th edn) (DSM-IV). Washington, DC: APA.

Baker, R.W., Tohen, M., Fawcett, J., et al (2003) Acute dysphoric mania: treatment response to olanzapine versus placebo. Journal of Clinical Psychopharmacology, 23, 132-137

Bauer, M. S., Whybrow, P. C., Gyulai, L., et al (1994) Testing definitions of dysphoric mania and hypomania: prevalence, clinical characteristics and inter-episode stability. Journal of Affective Disorders, 32, 201-2II. 
Cassidy, F. \& Carroll, B. J. (200I) Frequencies of signs and symptoms in mixed and pure episodes of mania: implications for the study of manic episodes. Progress in Neuropsychopharmacology and Biological Psychiatry, 25 659-665.

Cassidy, F., Murry, E., Forest, K., et al (1998) Signs and symptoms of mania in pure and mixed episodes. Journal of Affective Disorders, 50, |87-20|.

\section{Cassidy, F., Ahearn, E., Murry, E., et al (2000)}

Diagnostic depressive symptoms of the mixed bipolar episode. Psychological Medicine, 30, 403-4II.

Cohen, J. (1988) Statistical Power Analysis for the Behavioral Sciences. Hillsdale, NJ: Lawrence Erlbaum.

Dilsaver, S. C., Chen, Y. R., Shoaib, A. M., et al (1999) Phenomenology of mania: evidence for distinct depressed, dysphoric, and euphoric presentations. American Journal of Psychiatry, 156, 426-430.

First, M. B., Spitzer, R. L., Gibbon, M., et al (1997) Structured Clinical Interview for the Diagnostic and Statistical Manual, Fourth Edition, Patient Version. Washington, DC: American Psychiatric Press.

\section{Freeman, T.W., Clothier, J. L., Pazzaglia, P., et al} (1992) A double-blind comparison of valproate and lithium in the treatment of acute mania. American Journal of Psychiatry, 149, 108-1II.

Hamilton, M. (1967) Development of a rating scale for primary depressive illness. British Journal of Social and Clinical Psychology, 6, 278-296.

Himmelhoch, J. M. \& Garfinkel, M. E. (1986) Sourc of lithium resistance in mixed mania. Psychopharmacology Bulletin, 22, 613-620.

Himmelhoch, J. M., Mulla, D., Neil, J. F., et al (1976) Incidence and significance of mixed affective states in a bipolar population. Archives of General Psychiatry, 33 , 1062-1066.

Keller, M. B., Lavori, P. W., Coryell, W., et al (1986) Differential outcome of pure manic, mixed/cycling, and pure depressive episodes in patients with bipolar illness. JAMA, 255, 3138-3142.

Kraepelin, E. (192I) Manic-Depressive Insanity and Paranoia (ed. G. M. Robertson, trans. R. M. Barclay). Edinburgh: E\&S Livingstone.

Maier, W., Philipp, M. \& Gerken, A. (1985) Dimension of the Hamilton Depression Scale. Factor analysis studies. European Archives of Psychiatry and Neurological Science, 234, 417-422.

McElroy, S. L., Keck, P. E., Pope, H. G., et al (1988) Valproate in the treatment of rapid-cycling bipolar disorder. Journal of Clinical Psychopharmacology, $\mathbf{8}$, 275-279.

McElroy, S. L., Keck, P. E., Pope, H. G., et al (1992) Clinical and research implications of the diagnosis of dysphoric or mixed mania or hypomania. American Journal of Psychiatry, 149, 1633-1644.

Montgomery, S. A., Schatzberg, A. F., Guelfi, J. D., et al (2000) Pharmacotherapy of depression and mixed states in bipolar disorder. Journal of Affective Disorders, 59 (suppl. I), S39-S56.

Perugi, G., Akiskal, H. S., Micheli, C., et al (1997) Clinical subtypes of bipolar mixed states: validating a broader European definition in 143 cases. Journal of Affective Disorders, 43, 169-180.

Perugi, G., Akiskal, H. S., Micheli, C., et al (200I) Clinical characterization of depressive mixed state in bipolar-I patients: Pisa-San Diego collaboration. Journal of Affective Disorders, 67, 105-114.
Post, R. M., Rubinow, D. R., Uhde, T.W., et al (1989) Dysphoric mania. Clinical and biological correlates. Archives of General Psychiatry, 46, 353-358.

Secunda, S. K., Swann, A., Katz, M. M., et al (1987) Diagnosis and treatment of mixed mania. American Journal of Psychiatry, 144, 96-98.

Swann, A. C., Bowden, C. L., Morris, D., et al (1997) Depression during mania. Treatment response to lithium or divalproex. Archives of General Psychiatry, 54, 37-42.

Thase, M. E., Greenhouse, J. B., Frank, E., et al (1997) Treatment of major depression with psychotherapy or psychotherapy-pharmacotherapy combinations. Archives of General Psychiatry, 54, 1009-1015.

Tohen, M., Jacobs, T. G., Grundy, S. L., et al (2000) Efficacy of olanzapine in acute bipolar mania: a doubleblind, placebo-controlled study. The Olanzapine HGGW Study Group. Archives of General Psychiatry, 57, 84I-849.

Tohen, M., Chengappa, K. N., Suppes, T., et al (2002) Efficacy of olanzapine in combination with valproate or lithium in the treatment of mania in patients partially nonresponsive to valproate or lithium monotherapy Archives of General Psychiatry, 59, 62-69.

Tohen, M., Vieta, E., Calabrese, J., et al (2003a) Efficacy of olanzapine and olanzapine in combination with fluoxetine in the treatment of bipolar depression. Archives of General Psychiatry, 60, 1079-1088.

Tohen, M., Goldberg, J. F., Gonzalez-Pinto Arrillaga, A. M., et al (2003b) A I2-week, double-blind comparison of olanzapine vs. haloperidol in the treatment of acute mania. Archives of General Psychiatry, 60. $1218-1226$

Young, R. C., Biggs, J.T., Ziegler, V. E., et al (1978) A rating scale for mania: reliability, validity and sensitivity. British Journal of Psychiatry, 133, 429-435. 